\title{
Predictive factors in the outcome of pseudocysts complicating alcoholic chronic pancreatitis
}

B Gouyon, P Lévy, P Ruszniewski, M Zins, P Hammel, V Vilgrain, A Sauvanet, J Belghiti, P Bernades

\begin{abstract}
Aims-To determine the clinical, biochemical, and/or morphological features which could predict the need for treatment of pseudocysts at diagnosis in a homogeneous population of patients with alcoholic chronic pancreatitis.

Methods-Between January 1983 and December 1993, all patients followed for alcoholic chronic pancreatitis complicated by pseudocysts and confirmed by computed tomography (CT) scan at diagnosis were studied retrospectively. Two groups of pseudocysts were considered according to their pattern of evolution and the therapeutic requirements. Group I included 45 pseudocysts that regressed spontaneously ( 25 patients) or that persisted without symptoms (20 patients). Group II included 45 pseudocysts with persisting symptoms or complications, requiring surgical or non-surgical treatment. The evolution of pseudocysts was monitored by CT scanning or abdominal ultrasound. Initial CT scans of all patients were reviewed by an experienced radiologist. For each patient with pseudocysts, the following morphological parameters were recorded: number of pseudocysts, maximal diameter, location, intrapancreatic or extrapancreatic development, complications related to the pseudocyst, pancreatic calcifications, enlargement of the main pancreatic duct, and signs of recent acute pancreatitis. Univariate analysis, and then multivariate analysis with all significant variables on univariate analysis were performed.
\end{abstract}

Results-On univariate analysis, location of pseudocysts in the pancreatic head and intrapancreatic development of pseudocysts were significantly more frequent in group I than in group II $(78 \%$ versus $55 \%$, $\mathrm{p}<0.02$ and $89 \%$ versus $60 \%, \mathrm{p}<0.001$, respectively). The median diameter of pseudocysts was significantly smaller in group I than in group II (25 (10-110) $\mathrm{mm}$ and $40(10-120) \mathrm{mm}$ respectively, p<0.001). No differences between groups I and II were found for the clinical or biochemical parameters. Multivariate analysis showed that the intrapancreatic development of pseudocysts and a diameter less than $4 \mathrm{~cm}$ were the only independent factors associated with a spontaneous and favourable outcome. These factors accounted for $20 \%$ of the total variance.
Conclusions-Pseudocysts larger than $4 \mathrm{~cm}$ and extrapancreatic development can be considered independent predictive factors of persisting symptoms and/or complications in patients with pseudocysts and alcoholic chronic pancreatitis. (Gut 1997; 41: 821-825)

Keywords: chronic pancreatitis; pancreatic pseudocysts; computed tomography

Pseudocysts are encapsulated collections of pancreatic juice surrounded by a wall or capsule. They are a frequent complication of chronic pancreatitis. ${ }^{1-6}$ They may regress spontaneously, persist with or without symptoms, or result in complications. ${ }^{7-12}$ The predictive factors of these different outcomes, although obviously important in the management of patients with pseudocysts, are poorly understood. Available studies usually include both necrotic pseudocysts (those occurring after an attack of acute pancreatitis, due to the disruption of pancreatic duct integrity and the accumulation of pancreatic secretions in the interstices of the gland), and retentional pseudocysts which only occur during chronic pancreatitis, due to the coalescence of localised dilatations of the pancreatic ducts. However, since these two types of pseudocyst probably have different evolutive features, ${ }^{4}{ }^{1113-15}$ guidelines for pseudocyst management cannot be derived from these studies. Moreover, diagnostic criteria of pseudocysts vary widely in the same study. The development of abdominal ultrasound and computed tomography (CT) scanning has greatly improved the diagnosis and follow up of pseudocysts, providing better knowledge of their natural history. ${ }^{8116}$ The aim of the present study was to determine which clinical, biochemical, and/or morphological features at diagnosis were predictive of the need for treatment of pseudocysts (rather than no treatment) in a homogeneous population of patients with alcoholic chronic pancreatitis. Two groups of patients with pseudocysts were compared: those requiring medical or surgical intervention and those left untreated. Predictive factors for these therapeutic decisions were investigated by univariate and multivariate analysis.

\section{Patients and Methods}

INCLUSION CRITERIA

The study population included all patients who were followed for chronic alcoholic pancreatitis complicated by pseudocysts between January
Accepted for publication 23 June 1997 
1983 and December 1993 in the Departments of Gastroenterology and Surgery, at the Beaujon Hospital, Clichy, France. Only those pseudocysts identified by CT scanning at diagnosis were included in the study. Pseudocysts were only treated if they were the cause of symptoms or complications throughout the inclusion period.

\section{EXCLUSION CRITERIA}

Patients with pseudocysts were excluded from the study for the following reasons: no CT scan was performed within 15 days of the diagnosis of pseudocysts; the chronic pattern or the alcoholic origin of pancreatitis was not certain; another cause of pseudocysts (recent trauma, surgery, pancreatic cancer) was present; follow up of pseudocysts was less than 45 days; or surgery unrelated to pseudocysts was performed. Retrospective analysis of all patients included in this study was performed.

DEFINITIONS

The diagnosis of pseudocysts was based on CT findings. A pseudocyst was defined as a collection of liquid more than $10 \mathrm{~mm}$ in diameter, which was clearly distinct from the pancreatic parenchyma and the adjacent organs, and exhibited low density before intravenous injection of a contrast medium, which was not enhanced after injection. Multiple pseudocysts were defined as at least two such lesions.

Patients with pseudocysts were divided into two groups, based on the pattern of evolution of the disease and treatment. Group I included all cases that regressed spontaneously, or persisted without symptoms or treatment (except exploratory paracentesis). Group II included all cases requiring surgical or nonsurgical treatment (transparietal drainage, with or without aspiration).

The diagnosis of chronic pancreatitis was based on the presence of at least one of the following criteria: pancreatic calcifications on abdominal plain $x$ ray, CT scan, ultrasound, or endoscopic ultrasound; anomalies of the pancreatic ducts at pancreatic retrograde endoscopy, which were at least moderate according to the international Cambridge classification ${ }^{17}$; histological diagnosis of chronic pancreatitis with a biopsy or surgical specimen.

The alcoholic origin of chronic pancreatitis was defined as a daily alcohol intake of at least $60 \mathrm{~g}$ per day for at least two years, and when other aetiologies such as hypercalcaemia, hyperlipaemia, and hereditary or tropical pancreatitis had been excluded. Recent acute pancreatitis was defined as an episode of pancreatic pain requiring hospitalisation in the month preceding the diagnosis of pseudocysts, with raised serum pancreatic enzymes (amylase and/or lipase concentrations more than three times the upper limit of normal values) or radiological signs of acute pancreatitis on CT scan, grade $\mathrm{C}$ to $\mathrm{E}$ according to Balthazar's classification. ${ }^{18}$

If several pseudocysts occurred successively in the same patient, they were considered and analysed separately if at least one year had elapsed between two consecutive pseudocysts.

\section{DATA COLLECTION}

The first CT scan was considered to be the reference procedure in all patients. All CT scans were reviewed by the same author (MZ), who was unaware of the information on the patients' outcome. CT scans were performed before and after an intravenous injection of contrast medium, with thin or semi-thin slices (4-8 $\mathrm{mm})$. For each patient with pseudocysts, the following parameters were recorded: number of pseudocysts; maximal diameter (of the greatest pseudocyst if multiple); location (head, body, or tail of the pancreas, or adjacent organs); intrapancreatic or extrapancreatic development (a pseudocyst was considered intrapancreatic if it was surrounded by a continuous band of pancreatic parenchyma); complications related to pseudocysts (dilatation of intrahepatic or extrahepatic bile ducts, intracystic bleeding, portal, splenic or mesenteric vein thrombosis, haematoma or rupture of the spleen, or rupture of the pseudocyst). Furthermore, pancreatic calcifications, enlargement of the main pancreatic duct (more than $5 \mathrm{~mm}$ in the head and more than $3 \mathrm{~mm}$ in the body/tail of the pancreas), intraductal stones and signs of recent acute pancreatitis according to Balthazar's classification were also searched for.

The following clinical and biochemical data were recorded: symptoms related to pseudocysts (pain, fever, vomiting, abdominal mass); time elapsed from the diagnosis of chronic pancreatitis; recent acute pancreatitis which could account for the development of pseudocysts; inflammation tests (erythrocyte sedimentation rate and fibrinogen); pancreatic enzymes (serum lipase and amylase).

TREATMENT OF PSEUDOCYSTS

Surgical treatment of pseudocysts included pancreatic resection and pseudocyst drainage. Ultrasound or CT guided pseudocyst drainage was also performed. The indications for surgery were complications of pseudocysts in all cases, either acute (bleeding, infection, fistula, or rupture) or chronic (pain, digestive, or biliary compression), persisting after treatment of symptoms. Fine needle aspiration without drainage was considered a diagnostic, not a therapeutic procedure.

\section{PATIENT FOLLOW UP}

The evolution of pseudocysts was monitored by CT scan or abdominal ultrasound. The duration of follow up was defined as the duration of the time from diagnosis of pseudocysts to last imaging.

\section{STATISTICAL ANALYSIS}

Results were expressed as median and range. Univariate analysis was performed with the non-parametric Mann-Whitney $U$ test for quantitative data, and the exact Fisher's test for qualitative data. The level of statistical significance was set at $p=0.05$. Multivariate analysis was performed using step by step logistic regression. All significant variables at univariate analysis were considered for multivariate analysis. In a first analysis, the size of 
TABLE 1 Characteristics of patients with untreated (group I) and treated (group II) pancreatic pseudocysts

\begin{tabular}{|c|c|c|c|}
\hline & Group I $(n=45)$ & Group II $(n=45)$ & Fisher's test \\
\hline Median age (years) & $44(30-69)$ & $45(28-68)$ & NS \\
\hline Men $(\%)$ & 89 & 91 & NS \\
\hline $\begin{array}{l}\text { Median (range) duration (years) of alcohol } \\
\text { intake }\end{array}$ & $20(8-40)$ & $20(10-45)$ & NS \\
\hline $\begin{array}{l}\text { Median (range) age (years) at onset of } \\
\text { pancreatitis }\end{array}$ & $38(20-63)$ & $40(23-67)$ & NS \\
\hline $\begin{array}{l}\text { Median (range) time (months) from the first } \\
\text { symptom of chronic pancreatitis to the } \\
\text { diagnosis of pseudocyst }\end{array}$ & $15.1(0-254)$ & $24(0-177)$ & NS \\
\hline $\begin{array}{l}\text { Median (range) duration (months) of follow } \\
\text { up }\end{array}$ & $12.4(1.5-94)$ & $21.6(2-116)$ & $\mathrm{p}=0.02$ \\
\hline
\end{tabular}

TABLE 2 Number of patients with complicated untreated (group I) and treated (group II) pancreatic pseudocysts

\begin{tabular}{llll}
\hline & $\begin{array}{l}\text { Group I } \\
(n=45)\end{array}$ & $\begin{array}{l}\text { Group II } \\
(n=45)\end{array}$ & Fisher's test \\
\hline Acute complications & $\mathbf{1}$ & $\mathbf{8}$ & $\mathrm{p}=0.03$ \\
Infection & 0 & 2 & \\
Bleeding & 0 & 1 & \\
Pleural or bronchial fistula & 1 & 2 & \\
Rupture & 0 & 2 & \\
Haematoma of the spleen & 0 & 1 & $\mathrm{p}=0.003$ \\
Chronic complications & $\mathbf{1 3 \star}$ & $\mathbf{3 1}$ & \\
Bile duct dilatation & 5 & 13 & \\
Digestive compression & 3 & 11 & \\
Thrombosis of the the portal or splenic vein & 5 & 7 & $\mathrm{p}<0.001$ \\
Total & $\mathbf{1 4}$ & $\mathbf{3 9}$ & \\
\hline
\end{tabular}

${ }^{\star}$ Patients asymptomatic or with transient symptoms not requiring surgical intervention.

TABLE 3 Morphological characteristics of untreated (group I) and treated (group II) pancreatic pseudocysts, according to the initial CT scan

\begin{tabular}{llll}
\hline & Group I $(n=45)$ & Group II $(n=45)$ & Fisher's test \\
\hline Number of pseudocysts & & & \\
$\quad 1$ & 27 & 30 & $\mathrm{NS}$ \\
$\quad 18$ & 15 & $\mathrm{NS}$ \\
Location of pseudocysts & 35 & 25 & $\mathrm{p}<0.02$ \\
$\quad$ Head & 7 & 10 & $\mathrm{NS}$ \\
Body/tail & 2 & 3 & $\mathrm{NS}$ \\
Lesser sac & 1 & 7 & $\mathrm{NS}$ \\
$\quad$ Other & 40 & 27 & $\mathrm{p}<0.0001$ \\
Development of pseudocysts & 5 & 18 & $\mathrm{p}<0.0001$ \\
$\quad$ Intrapancreatic & & & \\
Extrapancreatic & & & \\
\hline
\end{tabular}

TABLE 4 Proportion of pancreatic pseudocysts above diameter thresholds according to treatment (untreated, group I; treated, group II)

\begin{tabular}{lllll}
\hline $\begin{array}{l}\text { Maximal diameter of } \\
\text { pseudocysts }\end{array}$ & $\begin{array}{l}\text { No of } \\
\text { pseudocysts }\end{array}$ & $\begin{array}{l}\text { GroupI } \\
(n=45)\end{array}$ & $\begin{array}{l}\text { Group II } \\
(n=45)\end{array}$ & Fisher's test \\
\hline$>2 \mathrm{~cm}$ & 77 & $44 \%(34)$ & $56 \%(43)$ & $\mathrm{p}=0.014$ \\
$>4 \mathrm{~cm}$ & 38 & $29 \%(11)$ & $71 \%(27)$ & $\mathrm{p}=0.0012$ \\
$>6 \mathrm{~cm}$ & 19 & $21 \%(4)$ & $79 \%(15)$ & $\mathrm{p}=0.009$ \\
$>8 \mathrm{~cm}$ & 14 & $29 \%(4)$ & $71 \%(10)$ & $\mathrm{NS}(\mathrm{p}=0.14)$ \\
\hline
\end{tabular}

pseudocysts was considered a quantitative variable. In a second analysis, a pseudocyst smaller than $4 \mathrm{~cm}$ in diameter was considered a qualitative variable.

\section{Results}

CLINICAL DATA

Ninety seven pseudocysts were considered in 92 patients. Seven patients were excluded because surgery unrelated to pseudocysts was performed (suspicion of pancreatic cancer in three patients, biliary or digestive compression in four patients). The study group thus included 90 pseudocysts in 85 patients (nine women, 76 men; median age 45 years).

Among the 90 pseudocysts, spontaneous regression or persistence without symptoms was observed in 25 and 20 cases, respectively. Group I thus included 45 pseudocysts (50\%) that did not require treatment. The size of the former 25 pseudocysts was not significantly different from the latter 20 pseudocysts (30 (15-80) and $20(10-110) \mathrm{mm}$ respectively, NS). Median time to regression of pseudocysts was 29 (2-143) weeks from diagnosis.

Group II included 45 pseudocysts (50\%) requiring therapeutic intervention-surgery (resection, drainage) in 42 patients, or radiological transcutaneous drainage in three patients.

Table 1 shows the characteristics of the populations of groups I and II. Table 2 lists the nature and distribution of complications related to pseudocysts. Most acute complications directly related to pseudocysts, such as infection, bleeding, rupture, or pancreatic fistula received emergency treatment and were included in group II. Biliary or digestive compression either regressed without treatment (group I), or necessitated treatment (group II).

Ultrasound and CT guided fine needle aspiration was performed in 31 patients-12 $(27 \%)$ and $19(42 \%)$ in groups I and II, respectively (NS). The procedure was indicated to check the relation between pseudocysts and pain and/or compression in all but six patients who were suspected of having infected pseudocysts.

Lasting regression of pseudocysts was not observed in any of the 31 patients. The following procedures were performed in the 42 group II patients operated on: internal drainage in 26 patients (cystogastric in 11, cystoduodenal in 10 , cystojejunal in five); distal splenopancreatectomy in 10 patients; and Whipple's procedure in six patients. In this group, regression of pseudocysts was obtained in $42 / 45$ cases (40 after surgery, two after transcutaneous drainage) after a median of $12(0-224)$ weeks. In the last three patients (two surgery, one transcutaneous drainage), no decrease in the size of pseudocysts was recorded, but all three patients became symptom free.

\section{PARAMETERS ASSOCIATED WITH THE OUTCOME} OF PSEUDOCYSTS

Univariate analysis

Table 3 shows the distribution of pseudocysts in groups I and II according to morphological criteria. The proportion of pseudocysts located in the pancreatic head, and of those with intrapancreatic development were significantly different between the two groups. The median size of pseudocysts was significantly smaller in group I than in group II $(25(10-110) \mathrm{mm}$ and $40(10-120) \mathrm{mm}$ respectively, $\mathrm{p}<0.001)$. Table 4 shows the distribution of pseudocysts according to different size thresholds.

No significant differences between groups I and II were found for the following parameters: calcifications in pancreatic parenchyma (32/45 versus $32 / 45$ ); enlargement of the main pancreatic duct (27/45 versus 26/45); stones in the main pancreatic duct (10/45 versus $15 / 45)$; and recent acute pancreatitis (21/45 versus 26/45). No significant differences in symptoms at time of diagnosis (pancreatic pain, vomiting, 
abdominal mass, fever $>38^{\circ} \mathrm{C}$, ascites, and pleural effusion) or in main blood tests at time of pseudocyst diagnosis (white blood cell count, erythrocyte sedimentation rate, serum fibrinogen, serum pancreatic enzymes, aminotransferases, alkaline phosphatase, and $\gamma$-glutamyl transferase) were found between groups I and II.

\section{Multivariate analysis}

Step by step logistic regression was performed based on the three factors significantly associated with the outcome of pseudocysts by univariate analysis. When the size of pseudocysts was considered as a quantitative variable (see Methods), the intrapancreatic development of pseudocysts was the only significant factor, accounting for $16 \%$ of the total variance $(p<0.001)$. When the qualitative criterion "pseudocysts less than $4 \mathrm{~cm}$ in diameter" was considered, it had independent prognostic value, accounting for $4 \%$ of the total variance $(\mathrm{p}<0.01)$. When considered together these factors accounted for $20 \%$ of the total variance.

\section{Discussion}

The identification of factors associated with the outcome of pseudocysts is of obvious clinical importance as pseudocysts may regress spontaneously or persist without symptoms, and not require treatment, or result in symptoms or complications that make treatment mandatory. ${ }^{19}$

The aim of this retrospective study was to determine which of the pseudocysts occurring during the course of alcoholic chronic pancreatitis warrant treatment. We therefore compared two large groups of pseudocysts: one group of 45 pseudocysts including all pseudocysts with a spontaneously favourable outcome; the other of 45 pseudocysts requiring treatment, due to persisting symptoms and/or complications.

Three of the morphological factors studied appeared to be associated with a spontaneouly favourable outcome by univariate analysis. Indeed, small sized pseudocysts, intrapancreatic pseudocysts, and pseudocysts located in the pancreatic head were likely to regress without treatment. However, many parameters were studied and statistical significance could have been reached by chance due to multiple comparisons. We thus performed multivariate analysis using the parameters selected during the first step. To our knowledge, this has not been performed previously to identify the factors associated with choice of treatment. Intrapancreatic pseudocysts, and pseudocysts smaller than $4 \mathrm{~cm}$ in diameter were the only independent factors accounting for $20 \%$ of the total variance.

The population in this study was as homogeneous as possible and multiple CT scans were performed in each patient for accurate comparisons. Most other studies have used several imaging techniques in the same patients including endoscopic retrograde cholangiopancreatography, ${ }^{50-24}$ intraoperative findings, ${ }^{4} 52122$ upper gastrointestinal barium series, ${ }^{13} 20212526$ angiography, ${ }^{212425}$ or clinical findings. ${ }^{23} 2526$ Imaging techniques with high sensitivity, such as abdominal ultrasound or CT scan, increase diagnostic accuracy. ${ }^{38112728}$ All our patients had confirmed chronic pancreatitis. Unlike the present study, in previous studies in the literature the aetiology of chronic pancreatitis has included alcoholic, hereditary, tropical, and hypercalcaemic pancreatitis. ${ }^{2122} 27$ Furthermore, few studies have included only pseudocysts occurring during chronic pancreatitis. ${ }^{522} 242729$

We observed spontaneous regression in $25.7 \%$ of pseudocysts. This figure cannot be compared with most series including pseudocysts complicating acute and chronic pancreatitis. Reported regression rates vary between $9 \%$ and $31 \%,{ }^{28911}$ mainly because of differences in inclusion criteria and the fact that most studies come from surgical series. Unlike Bradley et $a l,{ }^{8}$ who observed a short regression time for pseudocysts (less than six weeks in almost all cases), we found the median time to regression to be 29 weeks (range 2-143). Slow regression has also been reported by Vitas and Sarr ${ }^{10}$ in $7 / 13$ patients with spontaneous regression of pseudocysts, five of which regressed more than six months after diagnosis.

Persistent asymptomatic pseudocysts have been reported in $19 \%$ and $16 \%$ of patients by Yeo et $a l^{11}$ and Vitas and Sarr, ${ }^{10}$ respectively. In our study, 21 pseudocysts $(23 \%$ of the total population) remained asymptomatic.

The size of pseudocysts is usually considered to be closely related to their evolution. O'Malley et $a l^{4}$ observed regression in $55 \%$ of pseudocysts smaller than $4 \mathrm{~cm}$, with a $10 \%$ complication rate (versus $37 \%$ for pseudocysts larger than $4 \mathrm{~cm}$ ). In another study, regression was observed in $4 / 26(15.4 \%)$ pseudocysts larger than $6 \mathrm{~cm}$ and in 20/67 (34\%) pseudocysts smaller than $6 \mathrm{~cm} .{ }^{20}$ Finally, Yeo et $a l^{11}$ found that surgery was necessary for $67 \%$ of pseudocysts larger than $6 \mathrm{~cm}$ and for $40 \%$ of those which were smaller than $6 \mathrm{~cm}$. However, in a group of 114 pseudocysts, Vitas and Sarr ${ }^{10}$ showed that the size of pseudocysts was not significantly associated with their outcome. In the subgroup of 24 untreated patients with regular imaging follow up, that study showed that $80 \%$ of the pseudocysts less than $5 \mathrm{~cm}$, but also $50 \%$ of those greater than $5 \mathrm{~cm}$, regressed. ${ }^{10}$ It should be emphasised, however, that only one third of the patients in this study had pseudocysts related to chronic pancreatitis. Unlike these results, the present study shows that the initial size of pseudocysts is an important predictive factor for the necessity of treatment. Seventy one per cent of pseudocysts larger than $4 \mathrm{~cm}$ required treatment. There was no further significant increase in this rate for greater thresholds $79 \%$ and $71 \%$ for pseudocysts greater than $6 \mathrm{~cm}$ and $8 \mathrm{~cm}$, respectively). Thus a diameter less than $4 \mathrm{~cm}$ is associated with a favourable outcome as confirmed by multivariate analysis.

Pseudocysts occurring during chronic pancreatitis are located in the pancreatic head in $34.4-67.6 \%$ of patients. ${ }^{522427}$ In this study, $66 \%$ of pseudocysts were located in the head, and $57 \%$ of them did not require treatment. 
The location of pseudocysts was not an independent factor by multivariate analysis.

Intrapancreatic or extrapancreatic development of pseudocysts has rarely been studied in previous series. The rates of intrapancreatic pseudocysts were $50 \%, 65 \%$, and $99.4 \%$ in three studies, respectively ${ }^{51627}$ but the definition of these pseudocysts was not always provided. We defined an "intrapancreatic pseudocyst" as a pseudocyst completely surrounded by the pancreatic parenchyma, and found that $72.2 \%$ of pseudocysts were intrapancreatic, a feature clearly associated with a favourable spontaneous outcome (only $38.6 \%$ of these pseudocysts required treatment). This was confirmed by multivariate analysis.

It has been claimed that acute pseudocysts regress more frequently than chronic ones ${ }^{813} 30$ but this distinction is based on the time from diagnosis and not on the occurrence of recent acute pancreatitis. ${ }^{813}$ The proportion of pseudocysts due to acute pancreatitis during chronic pancreatitis is unknown. Recent acute pancreatitis was investigated in all our patients ${ }^{18}$ and found in 50.5\%. However, no difference between the two groups was observed and recent acute pancreatitis was not a factor associated with a favourable outcome in pseudocysts associated with chronic pancreatitis.

In conclusion, a diameter greater than $4 \mathrm{~cm}$ and extrapancreatic development can be considered independent predictive factors of persistent symptoms and/or complications in patients with pseudocysts and alcoholic chronic pancreatitis. These results can help clinicians select patients with pseudocysts requiring treatment.

1 Ammann RW, Akovbiantz A, Largiader F, Schueler G. Course and outcome of chronic pancreatitis: longitudinal study of a mixed medical-surgical series of 245 patients. Gastroenterology 1984; 86: 820-8.

2 Bernades P, Belghiti J, Athouel M, Mallardo N, Breil P, Fekete F. Histoire naturelle de la pancréatite chronique: étude de 120 cas. Gastroenterol Clin Biol 1983; 7: 8-13.

3 D'Egidio A, Schein M. Pancreatic pseudocysts: a proposed classification and its management implications. $\mathrm{Br} \mathcal{F}$ Surg 1991; 78: $981-4$.

4 O'Malley VP, Cannon JP, Postier RG. Pancreatic pseudocysts: cause, therapy, and results. Am F Surg 1985; 150: $680-2$.

5 Sarles JC, Salasc B, Delecourt P, Nacchiero M, Gaeta L. Les formations kystiques au cours des pancréatites chroniques: orientation thérapeutique. Gastroenterol Clin Biol 1982; 6: 857-62.
6 Staub JL, Le Genissel H, Sarles H. Etude de la sémiologie et des résultats du traitement chirurgical de 103 cas de pancréatite chronique compliqués de kystes ou de pseudokystes. Gastroenterol Clin Biol 1981; 5: 433-9.

7 Bradley EL, Clements LJ. Spontaneous resolution of pancreatic pseudocysts: implications for timing of operative intervention. Am f Surg 1975; 129: 23-8.

8 Bradley EL, Clements JL, Gonzales AC. The natural history of pancreatic pseudocysts: a unified concept of management. Am F Surg 1979; 137: 135-41.

9 Bourlières M, Sarles H. Pancreatic cysts and pseudocysts associated with acute and chronic pancreatitis. Dig Dis $S c i$ 1989; 34: 343-8.

10 Vitas GJ, Sarr MG. Selected management of pancreatic pseudocysts: operative versus expectant management. Surpseudocysts: operative vers
gery 1992; 111: 123-30.

11 Yeo CJ, Bastidas JA, Lynch-Nyhan J, Fishman EK, Zinner MJ, Cameron JL. The natural history of pancreatic MJ, Cameron JL. The natural history of pancreatic
pseudocysts documented by computed tomography. Surg

12 El Hamel A, Parc R, Adda G, Bouteloup PY, Huguet C, Malafosse M. Bleeding pseudocysts and pseudoaneurysms in chronic pancreatitis. Br F Surg 1991; 78: 1059-63.

13 Crass RA, Way LW. Acute and chronic pancreatic pseudocysts are different. Am F Surg 1981; 142: 660-3.

14 Andersson R, Janzon M, Sundberg I, Bengmark S. Management of pancreatic pseudocysts. Brf Surg 1989; 76: 550-2.

15 N'Guyen BLT, Thompson JS, Edney JA, Bragg LE, Rikkers LF. Influence of the etiology of pancreatitis on the natural history of pancreatic pseudocysts. Am F Surg 1991; 162: 527-31.

16 Luetmer PH, Stephens DH, Ward EM. Chronic pancreatitis: reassessment with current CT. Radiology 1989; 171: 353-7.

17 Sarner M, Cotton PB. Classification of pancreatitis. Gut 1984; 25: 756-9.

18 Balthazar EJ, Ranson JHC, Naidich DP, Megibow AJ, Caccavale R, Cooper MM. Acute pancreatitis: pronostic value of CT. Radiology 1985; 156:767-72.

19 Maule WF, Reber HA. Diagnosis and management of pancreatic pseudocysts, pancreatic ascites and pancreatic fistuas. In: Go VLW, Dimagno EP, Gardner JD, Lebenthal E, Reber HA, Scheele GA, eds. The pancreas: biology, pathobiology and diseases. New York: Raven Press, 1993; 741-50.

20 Sankaran S, Walt AJ. The natural and unnatural history of pancreatic pseudocysts. Br F Surg 1975; 62: 37-44.

21 Bodker A, Kjaergaard J, Schmidt A, Tilma A. Pancreatic pseudocysts: a follow-up study. Ann Surg 1981; 194: 80-4.

22 Kiviluoto T, Kivisaari L, Kivilaakso E, Lempinen M Pseudocysts in chronic pancreatitis: surgical results in 102 consecutive patients. Arch Surg 1989; 124: 240-3.

23 Beebe DS, Bubrik MP, Onstad GR, Hitchcock CR. Beebe DS, Bubrik MP, Onstad GR, Hitchcock CR. Management of pancreati

24 Etienne JC, Bouillot JL, Alexandre JH. Le traitement des formations kystiques des pancréatites chroniques: à propos de quarante et un cas. Ann Chir 1987; 41: 595-600.

25 Czaja AJ, Fisher M, Marin GA. Spontaneous resolution of pancreatic masses (pseudocysts?). Arch Intern Med 1975; 135: 558-62.

26 McConnel DB, Gregory JR, Sasaki TM, Vetto RM. Pancreatic pseudocyst. Am f Surg 1982; 143: 599-601.

27 Barthet M, Bugallo M, Moreira LS, Bastid C, Sastre B, Sahel J. Management of cysts and pseudocysts complicating chronic pancreatitis: a retrospective study of 143 patients. Gastroenterol Clin Biol 1993; 17: 270-6.

28 Aranha GV, Prinz RA, Esguerra AC, Greenlee HB. The nature and course of cystic pancreatic lesions diagnosed by ultrasound. Arch Surg 1983; 118: 486-8.

29 Munn JS, Aranha GV, Greenlee HB, Prinz RA. Simultaneous treatment of chronic pancreatitis and pancreatic pseudocyst. Arch Surg 1987; 122: 662-5.

30 Agha FP. Spontaneous resolution of acute pancreatic pseudocysts. Surg Gynecol Obstet 1984; 158: 22-6. 